\title{
Maternal serum and cord blood leptin concentrations at delivery in normal pregnancies and in pregnancies complicated by intrauterine growth restriction
}

Małgorzata Stefaniak $^{1 *}$, Ewa Dmoch-Gajzlerska ${ }^{1}$

Authors' Affiliations

${ }^{1}$ Department of Obstetrics and Gynecology Didactics, Faculty of Health Sciences, Medical University of Warsaw, Poland.

*Corresponding author:

E-mail: malgorzata.stefaniak@wum.edu.pl

Author Contributions:

Małgorzata Stefaniak - Conceptualization, Data curation, Formal analysis, Methodology, Writing original draft, Writing - review \& editing

Ewa Dmoch-Gajzlerska - Conceptualization, validation

Competing Interests: The authors have declared that no competing interests exist.

Financial Disclosure: The research was financed from the fund for scientific research and development projects selected on a highly competitive basis at the Faculty of Health Sciences, e Medical University of Warsaw (decision code: NZG/PM4/16).

The funders had no role in study design, data collection and analysis, decision to publish, or preparation of the manuscript.

Funding Information:

Medical University of Warsaw NZG/PM4/16 
Introduction: Leptin is a polypeptide hormone and in pregnancy it is secreted by the placenta and maternal and fetal adipose tissues. The expression of leptin and its specific receptors is observed in the uterine endometrium which indicates leptin involvement in the implantation process and embryonic/fetal development. Normal leptin production is a factor responsible for uncomplicated gestation, embryo development and fetal growth.

Objective: To compare at delivery maternal serum and cord blood leptin concentrations in normal pregnancies and in pregnancies complicated by intrauterine growth restriction (IUGR).

Material and methods: The study was performed in 25 pregnant women with isolated IUGR diagnosed by ultrasonography (study subjects) and in 194 pregnant women without any comorbid health conditions (controls). Leptin concentrations in maternal serum and in cord blood samples collected at delivery were measured by ELISA and subsequently analyzed by maternal Body Mass Index (BMI), mode of delivery, and infant gender and birth weight. For comparative analyses of normally distributed variables, parametric tests were used, i.e. the Student-t to test the assumption of homogeneity or non-homogeneity of variance and a One-Way ANOVA when more than two groups were compared. The non-parametric Mann-Whitney test was used when the distribution was not normal. The Pearson correlation coefficient was calculated to assess the correlation between normally distributed variables $(\mathrm{p}<0.05)$.

Results: In pregnancies complicated by IUGR, the mean maternal serum leptin concentration at delivery was significantly higher $(52.73 \pm 30.49 \mathrm{ng} / \mathrm{mL})$ than in normal pregnancies $(37.17 \pm 28.07 \mathrm{ng} / \mathrm{mL})$ $(\mathrm{p}=0.01)$. The mean cord blood leptin concentration in pregnancies complicated by IUGR was $7.97 \pm 4.46$ $\mathrm{ng} / \mathrm{mL}$ and significantly lower than in normal pregnancies $(14.78 \pm 15.97 \mathrm{ng} / \mathrm{mL})(\mathrm{p}=0.04)$. In normal pregnancies, but not in pregnancies complicated by IUGR, a statistically significant correlation was established between maternal serum leptin concentrations and maternal BMI at delivery $(\mathrm{r}=0,22 ; \mathrm{p}=0.00)$. No statistically significant correlation was found between cord blood leptin concentrations and maternal BMI in either study subjects or controls. In normal pregnancies, but not in pregnancies complicated by IUGR, a strong correlation was observed between cord blood leptin concentrations and birth weight $(\mathrm{r}=0,23 ; \mathrm{p}=0.00)$. In both study subjects and controls, there were no correlations between leptin concentrations in maternal serum and cord blood and infant gender and mode of delivery.

Conclusions: Elevated maternal blood leptin concentrations in pregnancies complicated by IUGR may indicate a significant adverse effect of elevated leptin on fetal growth. Enhanced leptin production by the placenta suggests leptin as a candidate marker of placental insufficiency. The differences in leptin concentrations, measured in maternal serum and in cord blood, between the study subjects and controls suggest that deregulated leptin levels may increase the risk of obstetric complications associated with placental insufficiency.

Key words: leptin, intrauterine growth restriction, birth weight, pregnancy, cord leptin 


\section{Introduction}

Extensive research into leptin conducted since its discovery over two decades ago has revealed its involvement in normal and pathological pregnancies [1]. It has been demonstrated that normal leptin production is a factor responsible for normal gestation, embryonic/fetal development and fetal growth. The presence of leptin in cord blood is another sign of its significant role in fetal development. Because of the pleiotropic mechanisms of its action and changes in concentrations throughout pregnancy, leptin levels may be considered a potential predictor for some complications of pregnancy, but this application requires further studies [1]. Leptin plays a key role in the earliest stages of pregnancy as it modulates the processes of proliferation, protein synthesis or placental apoptosis while the control of cellular proliferation is of significant importance for normal development of the placenta. In intrauterine growth restriction (IUGR), the structure of the blood vessel wall in the uterus and placenta is altered. The inner wall layers thicken and the lumen narrows which leads to changes in utero-placental blood flow. Decreases in circulating blood volume lead to increases in blood viscosity and vascular resistance which result in placental flow disorders [2]. With an insufficient placenta the exchange of nutrients between mother and developing fetus and adequate blood oxygenation are impaired. A reduced blood flow in the utero-placental and feto-placental circulation adversely affects the materno-fetal exchange and ultimately leads to chronic hypoxia and fetal growth arrest $[3,4]$. Intrauterine fetal growth is a multifactorial process determined by numerous genetic, metabolic and hormonal causes in mother, developing fetus and placental tissue. IUGR is a complex obstetric problem which results from these etiologic factors acting together [3]. Disturbance of fetal growth dynamics which do not correspond to gestational age can be hazardous to gestation and is in fact the second leading cause of perinatal mortality after prematurity. Studies of the role of leptin in reproduction stress that its normal concentration is associated with normal embryonic/fetal development while deregulated levels may be responsible for abnormal birth weight [1]. Many authors consider leptin to be a regulator of fetal growth [1,5]. Blood concentrations of leptin reflect maternal nutritional status and additionally when deregulated are a risk factor for IUGR [1]. Low leptin levels have been associated with disorders of fetal growth. A correlation between the maternal leptin concentration at delivery and the neonate's birth weight has been reported [6]. Research has confirmed that maternal serum leptin concentrations at delivery were significantly elevated and cord blood concentrations were lower in pregnancies complicated by IUGR compared to non-IUGR pregnancies [8-11]. This may suggest a compensatory mechanism by which small placentas produce more leptin [10]. It has been also suggested that leptin may be a marker of placental insufficiency, which is associated with increased placental leptin production. On the other hand, some authors did not find any significant differences in cord blood leptin concentrations between IUGR and normal pregnancies [12]. These inconsistent findings show that the association between IUGR and leptin concentrations remains to be clarified and further prospective studies are needed. To date, studies have been conducted in small groups of subjects and they did not involve concurrent leptin measurements in maternal serum at delivery and in cord blood in mother-infant pairs. Our study aims at filling this gap in knowledge.

\section{Objective}

To compare at delivery maternal serum and cord blood leptin concentrations in normal pregnancies and in pregnancies complicated by IUGR. 


\section{Material and methods}

The study included 25 pregnant patients with isolated IUGR and 194 normal pregnant women who served as controls and was conducted in the period between January 2015 and June 2017 in two first- and secondlevel hospitals in Warsaw. All women gave informed written consent to participate in the study. The study was approved by the Bioethics Committee at the Medical University of Warsaw (approval code: $\mathrm{KB} / 204 / 214)$.

IUGR was diagnosed by ultrasonography (abdominal circumference $(\mathrm{AC})$ below the $5^{\text {th }}$ percentile AC of corresponding gestational age) and by postnatal assessment of the neonate (birth weight below the $10^{\text {th }}$ percentile weight of corresponding gestational age) $[13,14]$. Maternal serum leptin concentrations were measured at delivery, in women with IUGR between gestation weeks 33 and 39 and in controls between gestation weeks 33 and 41. Multiple gestation, complications of pregnancy other than IUGR, maternal chronic disease and intrauterine fetal death were the exclusion criteria.

The study material consisted of leptin samples derived from two sources, placental leptin measured in maternal blood and fetal leptin measured in cord blood. Maternal blood samples $(9 \mathrm{~mL})$ were collected from an antecubital vein at delivery and fetal blood samples $(9 \mathrm{~mL})$ were collected from the umbilical vein immediately after delivery. After centrifugation of full blood, sera were stored at $-80^{\circ} \mathrm{C}$ until leptin measurement. Measurements were performed by the immunoenzymatic test ELISA using commercial kits (R\&D Systems Bio-Techne).

The following were recorded for each mother-infant pair: maternal BMI at the time of leptin measurement, gestation weeks at delivery, birth weight, mode of delivery and infant gender. BMI was calculated as weight in kilograms divided by height in meters squared. Standard anthropometric measurements of the infants were performed. The measurements of the body length and weight were precise to $0.1 \mathrm{~cm}$ and $0.1 \mathrm{~kg}$, respectively.

Statistical analysis was performed with STATISTICA PL package (StatSoft, Poland). Descriptive statistics were used to present descriptions of quantitative variables. For comparative analyses of normally distributed variables, parametric tests were used, i.e. the Student- $t$ to test the assumption of homogeneity or nonhomogeneity of variance and a One-Way ANOVA when more than two groups were compared. The nonparametric Mann-Whitney test was used when the distribution was not normal. The Pearson correlation coefficient was calculated to assess the correlation between normally distributed variables $(\mathrm{p}<0.05)$.

\section{Results}

The mean age of women was $30.0 \pm 5.2$ years in the control (non-IUGR) group and $31.0 \pm 3.3$ years in the study (IUGR) group. The mean BMI at delivery was $28.1 \pm 4.1 \mathrm{~kg}$ in the control group and $25.7 \pm 3.3 \mathrm{~kg}$ in the study group. The mean length of gestation was $38.9 \pm 1.1$ weeks in the control group and $37 \pm 1.3$ weeks in the study group. The mean birth weight and length of neonates was $3354 \pm 503.9 \mathrm{~g}$ and $54.9 \pm 2.7 \mathrm{~cm}$, respectively in the control group and $2440 \pm 497.6 \mathrm{~g}$ and $49 \pm 3.1 \mathrm{~cm}$, respectively in the study group (Table $1)$.

Table 1. Subject characteristics 


\begin{tabular}{|c|c|c|c|c|c|c|c|c|c|c|c|c|}
\hline & \multicolumn{6}{|c|}{ Controls $(\mathrm{N}=194)$} & \multicolumn{6}{|c|}{ IUGR (N=25) } \\
\hline & Mean & Min & Max & $\begin{array}{l}2^{\text {th }} \\
\text { quartile }\end{array}$ & $\begin{array}{l}75^{\text {th }} \\
\text { quartile }\end{array}$ & SD & Mean & Min & Max & $\begin{array}{l}25^{\text {th }} \\
\text { quartile }\end{array}$ & $\begin{array}{l}7^{\text {th }} \\
\text { quartile }\end{array}$ & SD \\
\hline $\begin{array}{l}\text { Maternal age } \\
\text { (years) }\end{array}$ & 30.0 & 18.0 & 44.0 & 26.0 & 34.0 & 5.2 & 31.0 & 25.0 & 37.0 & 30.0 & 34.0 & 3.3 \\
\hline $\begin{array}{l}\text { Third-trimester } \\
\text { BMI }\left(\mathrm{kg} / \mathrm{m}^{2}\right)^{*}\end{array}$ & 28.1 & 17.5 & 40.2 & 25.2 & 30.7 & 4.1 & 25.7 & 21.0 & 33.9 & 24.0 & 27.8 & 3.3 \\
\hline $\begin{array}{l}\text { Gestational age at } \\
\text { delivery (weeks) }\end{array}$ & 38.9 & 37.0 & 40.0 & 38.0 & 40.0 & 1.1 & 37.0 & 33.0 & 39.0 & 36.0 & 37.0 & 1.3 \\
\hline Birth weight $(\mathrm{g})$ & 3454.3 & 1480.0 & 5030.0 & 3190.0 & 3780.0 & 503.9 & 2440.0 & 1750.0 & 3770.0 & 2030.0 & 2650.0 & 497.6 \\
\hline Birth length $(\mathrm{cm})$ & 54.9 & 44.0 & 62.0 & 54.0 & 57.0 & 2.7 & 49.0 & 42.0 & 57.0 & 47.0 & 50.0 & 3.1 \\
\hline
\end{tabular}

* $\mathrm{kg} / \mathrm{m}^{2}=$ kilograms $/$ meters squared

The mean maternal serum leptin concentration in pregnancies with IUGR was $52.73 \pm 30.49 \mathrm{ng} / \mathrm{mL}$ and it was significantly higher, by $42 \%$, than in normal pregnancies $(\mathrm{p}=0.01)$. The mean cord blood leptin concentration in IUGR was $7.97 \pm 4.46 \mathrm{ng} / \mathrm{mL}$ and it was significantly lower, by $46 \%$, than in infants without IUGR ( $\mathrm{p}=0.04)$ (Table 2, Figs 1 and 2).

Table 2. Statistical analysis of the differences in leptin concentrations between normal pregnancies (controls) and pregnancies complicated by IUGR

\begin{tabular}{llllllllll}
\hline $\begin{array}{l}\text { Leptin } \\
\text { concentration }\end{array}$ & $\begin{array}{l}\text { Controls } \\
\text { Mean }\end{array}$ & $\begin{array}{l}\text { IUGR } \\
\text { Mean }\end{array}$ & $\mathrm{t}$ & $\mathrm{df}$ & $\mathrm{p}$ & $\begin{array}{l}\text { Controls } \\
\mathrm{N}\end{array}$ & $\begin{array}{l}\text { IUGR } \\
\mathrm{N}\end{array}$ & $\begin{array}{l}\text { Controls } \\
\text { SD }\end{array}$ & $\begin{array}{l}\text { SD } \\
\text { IUGR }\end{array}$ \\
\hline Maternal serum & $\mathbf{3 7 . 1 7}$ & $\mathbf{5 2 . 7 3}$ & $\mathbf{- 2 . 5 8}$ & $\mathbf{2 1 7}$ & $\mathbf{0 . 0 1}$ & $\mathbf{1 9 4}$ & $\mathbf{2 5}$ & $\mathbf{2 8 . 0 7}$ & $\mathbf{3 0 . 4 9}$ \\
\hline Cord blood & $\mathbf{1 4 . 7 8}$ & $\mathbf{7 . 9 7}$ & $\mathbf{2 . 1 2}$ & $\mathbf{2 1 7}$ & $\mathbf{0 . 0 4}$ & $\mathbf{1 9 4}$ & $\mathbf{2 5}$ & $\mathbf{1 5 . 9 7}$ & $\mathbf{4 . 4 6}$ \\
\hline
\end{tabular}

Abbreviations: $\mathrm{t}=$ Student's t-test; $\mathrm{df}=$ degree of freedom; $\mathrm{p}=\mathrm{p}$-value; $\mathrm{SD}=$ standard deviation

Fig. 1. The differences in maternal serum leptin concentrations between normal pregnancies (controls) and pregnancies complicated by IUGR (the median, quartiles and outliers)

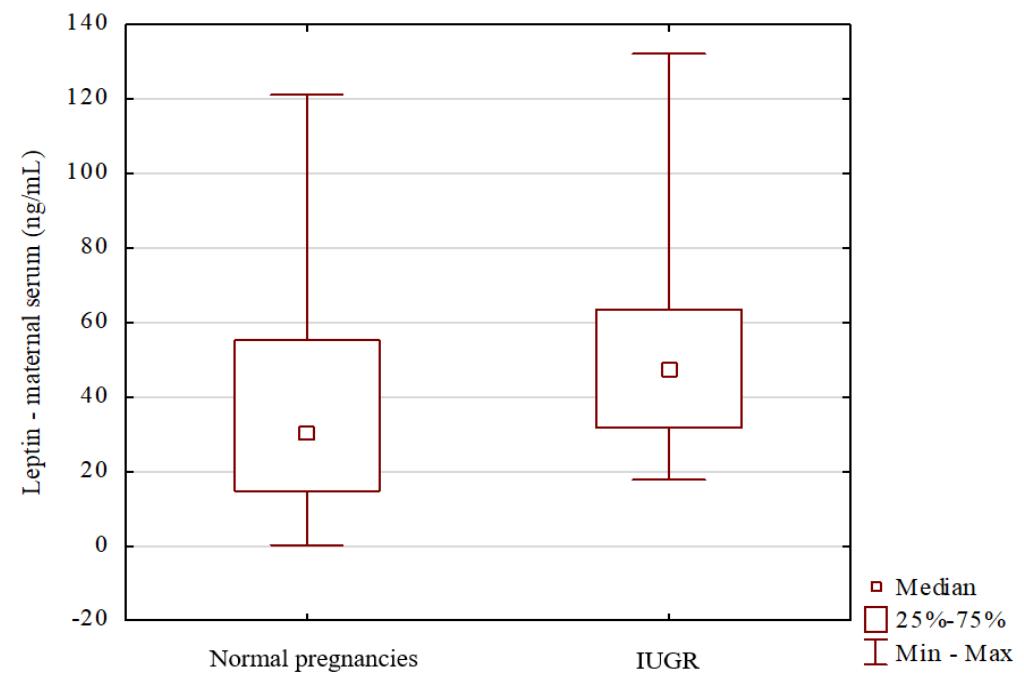


Fig. 2. The differences in cord blood leptin concentrations between normal pregnancies (controls) and pregnancies complicated by IUGR (the median, quartiles and outliers)

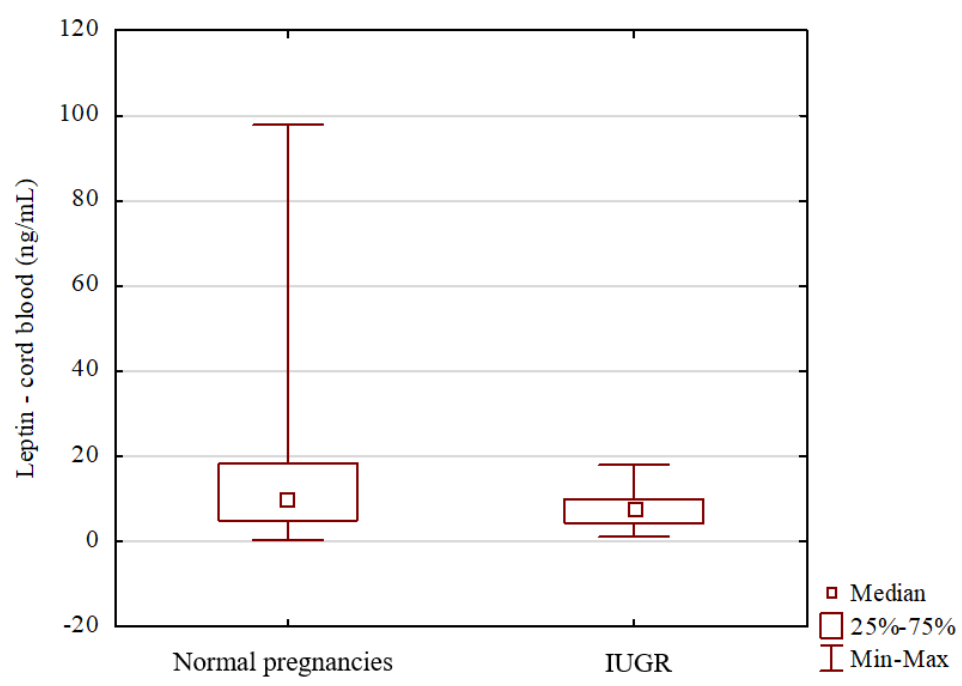

In women with normal pregnancies, a statistically significant positive correlation was established between maternal serum leptin concentrations and third-trimester BMI ( $\mathrm{r}=0.22 ; \mathrm{p}=0.00)$ (Fig. 3), but not between cord blood leptin and third-trimester BMI.

Fig. 3. Correlation between maternal serum leptin concentrations and third-trimester BMI 


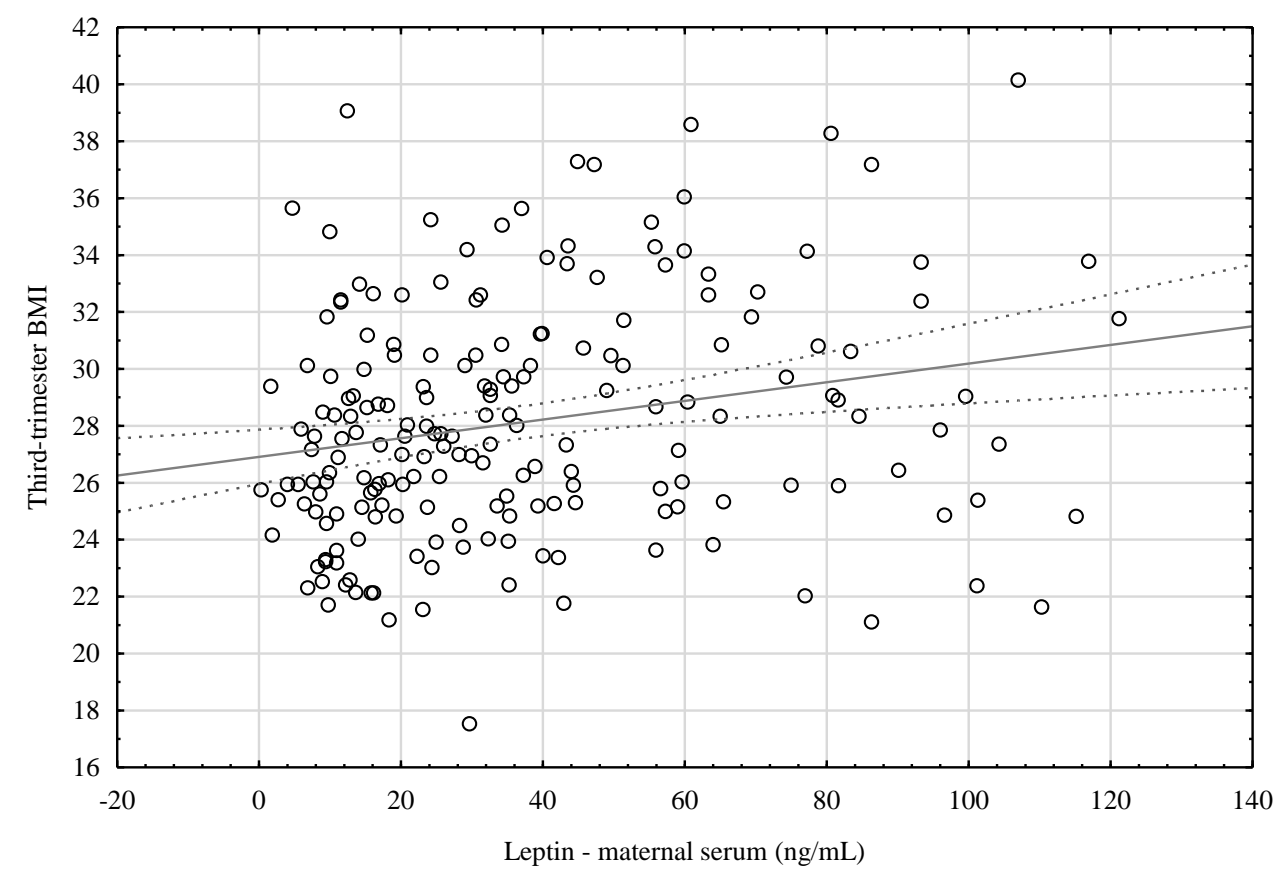

In women with pregnancies complicated by IUGR, no statistically significant correlations were established between third-trimester BMI and either maternal serum leptin or cord blood leptin.

In women with normal pregnancies, no statistically significant correlation was found between leptin concentrations in maternal serum and infant birth weight. However, a statistically significant positive correlation was established between leptin concentrations in cord blood and infant birth weight $(r=0.23$, $\mathrm{p}=0.00$ ) ( (Fig. 4). In pregnancies complicated by IUGR, no correlation was found between maternal serum and cord blood concentrations of leptin and infant birth weight.

Fig. 4. Correlation between cord blood leptin concentrations and infant birth weight $(r=0.23, p=0.00)$ 


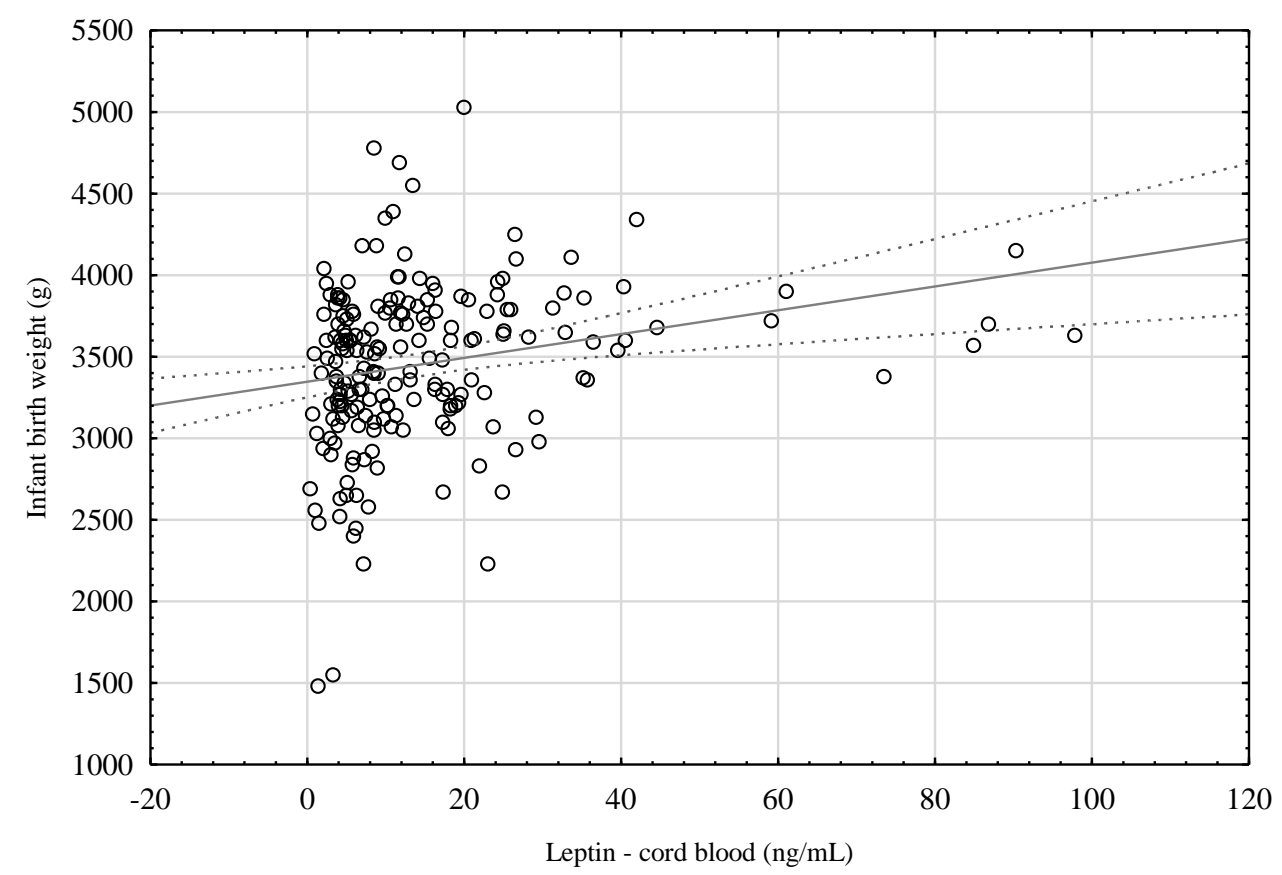

In both IUGR and non-IUGR pregnancies, there was no correlation between maternal serum and cord blood concentrations of leptin and infant gender or mode of delivery.

\section{Discussion}

Studies have demonstrated a significant role of normal leptin levels for fetal and neonatal growth and development and many authors believe that leptin actually acts as a regulator of fetal growth $[5,10,15$, 16]. This claim and opinion that leptin also is a marker of energy stores and a modulator of many biochemical processes during pregnancy is confirmed by the identification of leptin receptors in the placenta and in fetal tissues, including bone and cartilage [1]. Studies in pregnancies complicated by IUGR prove that placental abnormalities strongly correlate with low birth weight. Biswas et al. observed increased focal degeneration of the syncytiotrophoblast basal membrane in placentas from pregnancies complicated by IUGR compared to placentas from normal pregnancies [4]. Further observations demonstrated that chronic inflammation within the placental villi could be responsible for a significant reduction of the fetal-maternal exchange. Reduced transport of leptin gene transcriptions in the placentas of IUGR fetuses and low cord blood leptin concentrations have been reported. Catov et al. found lower leptin levels in neonates with IUGR diagnosed prenatally [8]. Studies in twin pregnancies found significantly lower cord blood concentrations of leptin in the twins with IUGR than in the co-twins with normal growth. In the study by Sooranna et al., the cord blood leptin concentrations in the twin with normal growth were at least two-fold higher than those in the co-twin with IUGR [9]. All these findings suggest a significant role of leptin as a regulator of fetal growth. In an earlier study, we established in normal pregnancies the reference intervals for leptin concentrations in the maternal serum and in cord blood. This study demonstrates significant differences in leptin concentrations, both in maternal serum and in cord blood, when measurements in normal pregnancies and in pregnancies complicated by IUGR are compared. In this study, we found that the mean maternal serum leptin concentration was significantly higher in pregnancies complicated by IUGR 
than in normal pregnancies. Comparison of the median leptin concentrations in maternal serum measured at delivery in the third trimester showed that in pregnancy weeks 36 to 39 the median maternal serum leptin levels in IUGR pregnancies were consistently higher than in normal pregnancies.The differences in leptin concentrations between normal and IUGR pregnancies suggest that leptin may have a role in the pathomechanism of some complications of pregnancy. Comparing leptin concentrations in IUGR pregnancies against the reference intervals established in normal pregnancies shows that leptin concentrations may be a useful prediction tool for complications of pregnancy. Semczuk-Sikora et al. reported similar findings of higher mean serum leptin levels in pregnant women with IUGR than in women with healthy pregnancies [6]. Elevated leptin levels may be maintained by an intrinsic compensatory mechanism as small placentas may produce more leptin in response to poor oxygen delivery. Our findings of significantly lower cord blood leptin concentrations in the infants with IUGR than in the infants with normal growth are in agreement with the results reported by Yildiz et al.[16] and Pighetti et al. [10]. Decreased cord blood concentrations of leptin in IUGR are thought to be related to reduced adipose tissue deposition in fetuses with IUGR and may signal long-lasting alterations in adipocyte function, possibly resulting in metabolic disorders in later life [15]. Some authors believe that IUGR is strongly associated with maternal nutrition at specific stages of gestation $[18,19]$. Maternal obesity carries an increased risk of complications in pregnancy including IUGR $[18,19]$. On the other hand, studies in hypotrophic neonates found that low-birth-weight infants were more frequently born to mothers who had protein- and calorierestricted $(600-900 \mathrm{kcal} /$ day $)$ diets in the second and third trimesters. Pre-pregnancy maternal body weight and its gain during pregnancy are the most important factors correlated with the infant's birth weight. However, maternal body weight is not a sole or most important risk factor for IUGR as the etiologies are diverse. Restricted maternal diets, e.g. glucose restriction, may result in fetal hypotrophy as glucose is the main nutrient necessary for fetal growth [2]. Accordingly, research was conducted to investigate any possible relationship between maternal leptin levels, BMI and glucose levels and to find out whether disturbances in carbohydrate metabolism could be another risk factor for IUGR. Like Pighetti et al. [10] we did not find any significant correlations between BMI and leptin concentrations in either maternal serum or cord blood in pregnancies complicated by IUGR, but in non-IUGR pregnancies a significant correlation was established between maternal BMI and serum leptin levels. Marino-Ortega et al. reported similar findings and demonstrated a strong association between blood leptin concentrations and weight gain in pregnancy [26]. Schroff et al. described a moderate correlation between maternal blood leptin concentrations and BMI [15]. Yildz et al. found a positive correlation between maternal serum leptin concentrations and BMI in pregnancies with IUGR [16]. These discrepancies in the results reported from studies in IUGR and non-IUGR pregnancies show that the relationship between BMI and maternal blood leptin concentrations has not been fully elucidated and maternal leptin levels could be influenced by other factors or complications of pregnancy. Studies in premature and small for gestational age infants with low birth weight found lower cord blood concentrations than in infants with high birth weight [21-25]. The authors argue that in utero leptin acts as a regulator of neuronal circuits controlling nutrient intake and appetite. Leptin not only regulates body weight but also has an important role in newborn adaptation to extrauterine life. The level of neonatal stress depends on such factors as gestational age, birth weight or mode of delivery. Leptin reduces neonatal responsivity to stress by an increased expression of glucocorticoid receptors in the central nervous system and an increased sensitivity to glucocorticoid negative feedback [26]. In this study, no correlation was established between birth weight and maternal serum leptin levels in pregnancies complicated by IUGR, which was in agreement with the results reported by Pighetti et al. [10]. These findings may confirm the hypothesis that placental and fetal leptins act individually. Lower leptin 
concentrations in cord blood may suggest that it is secreted mainly by fetal adipose tissue rather than the placenta [10]. Also Yildiz et al. [16] did not find any correlation between maternal serum leptin concentrations and infant birth weight, although a correlation between cord blood leptin and infant birth weight, but not infant BMI, was established by Pighetti et al. [10] and Tamura et al. [27]. These observations suggest that leptin is not a sole regulator of intrauterine growth disorders. Most published studies in IUGR pregnancies do not describe any correlation between maternal blood leptin concentrations and fetal weight [27-29]. Since no significant correlations have been established and the results reported from different centers are inconsistent, the relationship between leptin levels and fetal growth/development obviously needs further studies. In this study, we also assessed maternal serum leptin concentrations and infant birth weight in normal pregnancies and found no statistically significant correlation. However, there was a statistically significant correlation between cord blood leptin concentrations and birth weight. The findings are confirmed in the literature and attributed to the role played by leptin in the regulation of appetite and metabolism. Leptin may be responsible for body weight changes in neonates in the first days after birth and its levels may serve as an indicator of nutritional status [8,22-25,30,31].

\section{Strengths and limitations}

The presented results have certain limitations which should be taken into account in their analysis and interpretation. This was a cross-sectional study and the study group is relatively small. However, considering that in pregnancies complicated by IUGR we found significantly higher maternal serum leptin concentrations than in normal pregnancies, it may be assumed that maternal blood leptin could be a valuable additional marker (diagnostic tool) to use for predicting some complications of pregnancy. Before its clinical use could be reliably recommended, further studies and clinical observations are needed. It would be advisable to check the reproducibility and generalizability of the present results in cohort studies and in prospective studies in larger populations of women recruited in earlier stages of pregnancy, to assess leptin levels for changes over time. Another important question deserving research is the relationship, if any, of low blood cord leptin concentrations in infants with IUGR and their further weight gain as they get older.

\section{Conclusions:}

1. Maternal serum leptin concentrations were elevated in pregnancies complicated by IUGR

2. In pregnancies complicated by IUGR, significant differences in leptin concentrations between maternal serum and cord blood may suggest the effect of an intrinsic compensatory mechanism whereby small placentas release larger amounts of leptin.

3. Prospectively, leptin can be assessed as a marker of placental insufficiency in complications of pregnancy other than IUGR.

4. Impaired leptin production was found in infants with IUGR compared to infants in normal pregnancies.

5. Low leptin concentrations in the cord blood of infants with IUGR indicate lower adipose tissue deposition and point to leptin involvement in the pathomechanism of IUGR. 
References

1.Pérez-Pérez A, Toro A, Vilariño-García T i wsp.: Leptin action in normal and pathological pregnancies. J Cell Mol Med. 2018; 22(2):716-727.

2.Baschat AA, Galan HL, Gabbe SG.: Intrauterine inhibition of fetal growth. w: Obstetrics. Normal and complicated pregnancy. (ed. Gabbe SG. et al.). Elsevier Urban \& Partner, Wrocław 2014; 625-626.

3.Suhag A, Berghella V.: Intrauterine Growth Restriction (IUGR): Etiology and Diagnosis. Curr Obstet Gynecol Rep. 2013; 2(2):102-111.

4.Biswas S, Ghosh SK.: Gross morphological changes of placentas associated with intrauterine growth restriction of fetuses: a case control study. Early Hum Dev. 2008; 84(6):357-362.

5.Perez-Perez A, Sanchez-Jimenez F, Maymo J i wsp.: Role of leptin in female reproduction. Clin Chem Lab Med. 2015; 53(1):15-28.

6.Semczuk-Sikora A, Krzyżanowski A, Semczuk M i wsp.: Maternal serum leptin levels in pregnancies complicated by preeclampsia or intrauterine growth restriction. Probl Hig Epidemiol. 2011; 92(3):504507.

7.Ashworth CJ, Hoggard N, Thomas L, Mercer JG, Wallace JM, Lea RG.: Placental leptin. Rev Reprod. 2000; 5(1):18-24.

8.Catov JM, Patrick TE, Powers RW, Ness RB, Harger G, Roberts JM.: Maternal leptin across pregnancy in women with small-for-gestational-age infants. Am J Obstet Gynecol. 2007; 196(6):558.e1-8.

9.Sooranna SR, Ward S, Bajoria R.: Fetal leptin influences birth weight in twins with discordant growth. Pediatr Res. 2001; 49(5):667-672.

10.Pighetti M, Tommaselli GA, D'Elia A i wsp.: Maternal serum and umbilical cord blood leptin concentrations with fetal growth restriction. Obstet Gynecol. 2003; 102(3):535-543.

11.Milenković S, Jankovic B, Mirković L, Jovandaric MZ, Milenković D, Otašević B1.: Lipids and adipokines in cord blood and at $72 \mathrm{~h}$ in discordant dichorionic twins. Fetal Pediatr Pathol. 2017; 36(2):106-122.

12.Aydin HI, Eser A, Kaygusuz I i wsp.: Adipokine, adropin and endothelin-1 levels in intrauterine growth restricted neonates and their mothers. J Perinat Med. 2016; 44(6):669-676.

13.Copel JA, Bahtiyar MO. A practical approach to fetal growth restriction. Obstet Gynecol. 2014; 123(5): 1057-1069;

14.Unterscheider J, Daly S, Geary MP, et al. Optimizing the definition of intrauterine growth restriction: the multicenter prospective PORTO Study. Am J Obstet Gynecol. 2013; 208(4): 290.e1-290.e6 
15.Shroff MR, Holzman C, Tian Y, Evans RW, Sikorskii A.: Mid-pregnancy maternal leptin levels, birthweight for gestational age and preterm delivery. Clin Endocrinol (Oxf). 2013; 78(4):607-613.

16. Yildiz L, Avci B, Ingeç M.: Umbilical cord and maternal blood leptin concentrations in intrauterine growth retardation. Clin Chem Lab Med. 2002; 40(11):1114-1117.

17.Shekawat PS, Garland JS, Shivpuri C i wsp.: Neonatal cord blood leptin: Its relationship to birth weight, body mass index, maternal diabetes, and steroids. Pediatr Res. 1998; 43(3):338-343.

18.Guzik TJ, Mangalat D, Korbut R.: Adipocytokines - novel link between inflammation and vascular function? J Physiol Pharmacol. 2006; 57(4):5028-5065.

19.Matarese G, Moschos S, Mantzoros Ch.: Leptin in immunology. J Immunol. 2005; 15:174(6):31373142.

20.Zhang Y, Proenca R, Maffei M, Barone M, Leopold L, Friedman JM.: Positional cloning of the mouse obese gene and its human homologue. Nature. 1994; 1:372(6505):425-432.

21.Sadownik B.: Relationships Between Leptin Concentration and Sex, Type of Delivery and Body Weight of Newborns During First Days of Life. Post Neonatol. 2007; 1:35-39.

22.Kirel B, Tekin N, Tekin B, Kiliç FS, Doğruel N, Aydoğdu SD.: Cord blood leptin levels: relationship to body weight, body mass index, sex and insulin and cortisol levels of maternal-newborn pairs at delivery. J Pediatr Endocrinol Metab. 2000; 13(1):71-77.

23.Su PH, Wang SL, Chen JY, Lai CP, Jian SH.: Serum leptin levels in preterm, healthy and sick-term newborns. Acta Paediatr Taiwan. 2002; 43(5):249-254.

24.Garanty-Bogacka B, Czeszyńska MB, Syrenicz M.: Immaturity or hypotrophy? The cord blood leptin levels in preterm and small for gestational age neonates. Gin Pol. 2003; 74:356-361.

25.Po-Jung Tsai, Chun-Hsien Yu, Shih-Penn Hsu; Cord plasma concentrations of adiponectin and leptin in healthy term neonates: positive correlation with birthweight and neonatal adiposity. Clin Endocrinol (Oxf). 2004; 61(1):88-93.

26.Marino-Ortega LA, Molina-Bello A, Polanco-García JC i wsp.: Correlation of leptin and soluble leptin receptor levels with anthropometric parameters in mother-newborn pairs. Int J Clin Exp Med. 2015; 15:8(7):11260-11267.

27.Tamura T, Goldenberg RL, Johnston KE, Cliver SP.: Serum leptin concentrations during pregnancy and their relationship to fetal growth. Obstet Gynecol. 1998; 91(3):389-395.

28.Miehle K, Stepan H, Fasshauer M.: Leptin, adiponectin and other adipokines in gestational diabetes mellitus and pre-eclampsia. Clin Endocrinol. 2012; 76(1):2-11.

29.Grisaru-Granovsky S, Eitan R, Algur N, Schimmel MS, Diamant YZ, Samueloff A.: Maternal and umbilical cord serum leptin concentrations in small-for-gestational-age and in appropriate-for-gestationalage neonates: a maternal, fetal, or placental contribution? Biol Neonate. 2003; 84(1):67-72. 
30.Bury A, Kulik-Rechberger B.: Relationships Between Leptin Concentration and Sex, Type of Delivery and Body Weight of Newborns During First Days of Life. Endokrynol Ped. 2011; 10(3):29-38.

31.Valūniene M, Verkauskiene R, Boguszewski M i wsp.: Leptin levels at birth and in early postnatal life in small- and appropriate-for-gestational-age infants. Medicina (Kaunas). 2007; 43(10):784-791. 